\title{
Comparative analysis of kinesiotherapy rehabilitation after hip arthroscopy, quantified by harris and vail hip scores: a retrospective study
}

\author{
Melissa Saavedra ${ }^{1}$ \\ Ricardo Moraga ${ }^{1}$ \\ Patricia Diaz ${ }^{1}$ \\ Daniel Camacho \\ Rodrigo Mardones ${ }^{2}$ \\ 1 Sports Medicine Center, Clínica Las Condes, Chile \\ 2 Articular Reconstructive Surgery Hip / Knee; Hip \\ arthroscopy Department of Orthopedics Clinica \\ Las Condes, Santiago de Chile, Chile
}

Corresponding author:

Rodrigo Mardones

Articular Reconstructive Surgery Hip / Knee

Hip arthroscopy Department of Orthopedics, Clinica

Las Condes

La Fontecilla 441, Las Condes, Santiago de Chile, Chile

E-mail:rmardones@clc.cl

\section{Summary}

Background: It has been proven that femoroacetabular impingement cases improve following arthroscopic surgery. However, rehabilitation has a major role in the patient's recovery. The protocol used by our institution consists of an evidence-based guideline for the different phases of rehabilitation.

Objective: Describe and determine the effectiveness of our institution's kinesiotherapy rehabilitation program during 2011-2016, comparing Harris and Vail Hip Scores scales (HHS and VHS, respectively) at the beginning of each treatment phase.

Materials and methods: This is an observational, descriptive, longitudinal and retrospective study which, from a total of 684 subjects who underwent surgery, and 103 subjects who followed our institution's rehabilitation program, ultimately uses a sample of 48 subjects for analysis; these subjects were included because they completed the scales on the three occasions determined.

Results: Significant exact differences were found in: multivariate contrasts HHS ( $F=147.420$; $\mathrm{p}=0.000)$; VHS $(\mathrm{F}=82,160 ; p=0,000)$. Mauchly's sphericity test: HHS $(W=0.722 ; p=0.001)$; VHS $(W=0.830 ; p=0.014)$. The within-subject effect showed significant exact differences in: HHS ( $F=169.451 ; p=0.000)$; VHS ( $F=115.387 ; p=0.000)$. Conclusion: Results showed significant exact differences $p=0.00$. In spite of its limitations, this study provides a guideline for a patient's safe return to daily life activities.

Level of evidence: IV.

KEY WORDS: hip arthroscopy, kinesiotherapy rehabilitation, Harris Hip score, Vail Hip score.

\section{Introduction}

Femoroacetabular impingement is nowadays known as one of the causes of pain, dysfunction, and responsible of degenerative articular changes in the population ${ }^{1,2}$. In conjunction with each person's anatomical alterations, this can trigger labral lesions and long-term degenerative changes, such as hip osteoarthritis ${ }^{1-3}$.

There are three types of femoroacetabular impingement: the Cam type, which manifests mostly in young males ${ }^{1}$; the Pincer type, which appears mostly in during middle age and is prevalent in females ${ }^{1}$; and the mixed type, which is a combination of Cam and Pincer $^{4}$.

The traditional or surgical treatment for this condition is very important in improving the patient's quality of life, as well as decreasing his or her symptoms and reducing the physical and emotional impact in the short and medium term ${ }^{1,2}$.

Hip arthroscopy surgery has proven to be an effective treatment as well as less invasive, which leads to a rapid rehabilitation diminishing symptoms and improving joint ranges of movement ${ }^{4,5}$.

The aim of post-surgery rehabilitation is the patient's functional recovery, the decrease of post-surgery symptoms and the improvement of the patient's quality of life.

Such rehabilitation must consider tissue healing, avoid capsular adhesions, and customize exercises according to the abilities of the patient, while at the same time complying with the exercises related to each rehabilitation phase ${ }^{6}$. The literature describes 4 to 5 rehabilitation phases, which go from maximum protection and mobility to functional return to sport activities 6,7

In addition, there are self-report scales which typify different aspects of daily life activities, symptomatology and personal perceptions, and are used in vari- 
Comparative analysis of kinesiotherapy rehabilitation after hip arthroscopy, quantified by harris and vail hip scores: a retrospective study

ous studies to differentiate a patient's progress before and after surgery. The Harris Hip score (HHS) is a reliable and valid tool used as self-perception instrument 8,9 . A study conducted by Hoeksma et al. (2003) showed that HHS had high sensitivity and specificity for hip osteoarthritis patients, as it showed high sensitivity in the assessment of participants' speed of gait, pain, and functionality in comparison to other self-report tools ${ }^{10}$

The literature shows that the HHS measuring scale can be used for various hip pathologies, and joint range is a critical measurement point, as long as it is evaluated before and after treatment ${ }^{11}$.

On the other hand, the Vail Hip Score (VHS) is a valid and reliable measurement scale which has been adapted for Spanish speakers to be used as self-report tool ${ }^{12}$.

The purpose of this study is to describe and determine the effectiveness of our institution's kinesiotherapy rehabilitation program between 2011-2016, comparing the scores obtained from the HHS and VHS self-report scales before treatment, at the 10th session and at the 20th session of treatment.

\section{Methodology}

\section{Design}

This study has an observational, descriptive, longitudinal and retrospective nature, using a non-probabilistic convenience sample. We analyzed Excel data of the HHS and VHS self-report scales of subjects who underwent hip arthroscopies in our institution.
From a total of 684 hip surgeries, 103 subjects entered our kinesiotherapy rehabilitation program (average age $=38.078$ ), from which 48 subjects (average age $=37.08$ ) were considered for the statistical analysis, as they met the following criteria for inclusion and exclusion: 1. They each underwent hip arthroscopy and subsequently entered rehabilitation in our institution. 2. They completed the HHS and VHS self-report scales before starting treatment, at the 10th session and at the 20th session.

Patients who did not complete the self-report questionnaires at the start of treatment, at the 10th session and at the 20th session of kinesiotherapy were excluded from the study.

\section{Instruments}

\section{Description of post-surgery protocol for hip impingement (Table I) \\ Phase 1 \\ Tissue protection, mobility and motor control phase (1-4 weeks post-surgery)}

The main objective of Phase 1 of rehabilitation immediately after arthroscopic hip surgery is protecting scar tissue and restoring independent mobility.

In order to achieve these goals during early rehabilitation, it is critical to focus on managing the acute inflammatory process, establishing independent gait with technical assistance, and initiating early range of motion $^{13}$. This is the phase of greater care, in which the guidelines and projections the rehabilitation are set. The importance lies in recognizing and managing

Table I. The rehabilitation protocol after hip arthroscopy for femoroacetabular impingement consists into 4 phases. The objective of the physiotherapy and the progression criteria of each phase are summarized into this Table.

\begin{tabular}{|c|c|c|}
\hline & OBJECTIVES & PROGRESSION CRITERIA \\
\hline $\begin{array}{l}\text { PHASE } 1 \\
1 \text { to } 4 \text { WEEKS }\end{array}$ & $\begin{array}{l}\text { The main objective in the first phase } \\
\text { of rehabilitation immediately after } \\
\text { arthroscopic hip surgery, is the protect } \\
\text { ion of scar tissue and restoration of } \\
\text { independent mobility. }\end{array}$ & $\begin{array}{l}\text { 1. Minimum Pain with the exercises of Phase } 1 . \\
\text { 2. Increased ROM. } \\
\text { 3. Muscle Activation adequatein all exercises. } \\
\text { 4. Remove canes }\end{array}$ \\
\hline $\begin{array}{l}\text { PHASE } 2 \\
4 \text { to } 8 \text { WEEKS }\end{array}$ & $\begin{array}{l}\text { The overall objective of this phase is } \\
\text { to perform activities of daily living } \\
\text { independently and pain-free manner. }\end{array}$ & $\begin{array}{l}\text { 1. Normal gait without pain } \\
\text { 2. Full range of motion } \\
\text { 3. No joint swelling, muscle pain or irritation } \\
\text { 4. Adequate neuromuscular control in functional } \\
\text { activities }\end{array}$ \\
\hline $\begin{array}{l}\text { PHASE } 3 \\
8 \text { to } 12 \text { WEEKS }\end{array}$ & $\begin{array}{l}\text { Phase } 3 \text { typically begins between } 6 \text { th } \\
\text { to } 8 \text { th postoperative week, depending } \\
\text { on the patient, their goal is to help } \\
\text { restore even more endurance and } \\
\text { muscle strength, improve cardiovascula } \\
\text { fitness, optimize neuromuscular control, } \\
\text { balance and proprioception. }\end{array}$ & $\begin{array}{l}\text { 1. The patient performs all exercises Phase } 3 \\
\text { painlessly and properly. } \\
\text { 2. Cardiovascular capacity } \\
\text { similar to preoperative. Step } \\
\text { 3. Test the Sport Hip only for medical reasons }\end{array}$ \\
\hline $\begin{array}{l}\text { PHASE } 4 \\
\text { SPORTS RETURN }\end{array}$ & Phase 4 aims to return to competition. & $\begin{array}{l}\text { Phase } 4 \text { aims to return to competition precautions to } \\
\text { consider at this stage include all activities must be } \\
\text { able to perform pain-free. Usually there are no specific } \\
\text { limitations, except that start with impact activities } \\
\text { initiated after the 5th month }\end{array}$ \\
\hline
\end{tabular}


posture and movements that have greatest impact on tissues: by doing this we can protect tissue during healing process. This has to be recognized on early stages of rehabilitation and has to be communicated to the subject in the clearest way possible in order to prevent complications. The information given to the subject is critical as he or she protects healing tissues during daily life activities.

In addition, it is important to control pain and inflammation through the use of physical agents and physiotherapy, which are a daily help in achieving goals for both the therapist and the subject.

The joint range of motion (ROM) is one of the priorities for the rehabilitation, and is based on repair rates for bone tissue, labral tissue, capsule-ligament tissue and cartilage. The main objective is to optimize tissue flexibility and minimize the risk of damage ${ }^{14}$.

Authors recommend avoiding excessive flexing, abduction, internal rotation, or any movement that may lead to increased inflammation and/or prolonged discomfort; by doing this we also avoid capsular adhesions, thus showing many similarities with shoulder post-surgery treatments ${ }^{15,16}$. Other major objectives of Phase 1 include early muscle activation, and medium intensity isometric exercises for thighs, pelvis and trunk musculature, which should start during the first week post-surgery, in order to delay muscle atrophy and prevent the effects of immobilization. Emphasis should be put on central and deep muscles, including the transversus abdominis and multifidus muscles, which promote stability in the lumbar spine during movement and bed mobility. It has been proved that individuals with no lower back pain who work activation of the transversus abdominis muscles through feed forward exercises show better results during hip rehabilitation $^{13}$.

Recurring findings of studies on hip arthroscopy postsurgeries relate to the loss of neuromuscular control. It is therefore one of the main points of focus during this phase: there is a focus on the recovery of neuromuscular control of the iliopsoas, tensor fasciae latae (TFL) and rectus femoris muscles, which have a tendency to hyperactivation, as well as other muscle groups like the gluteus medius and gluteus maximus, which present inhibition patterns; these groups of muscle have key roles in both hip movement and stability. Having this considered, emphasis has been made on the specific task of the hip abductors, particularly the gluteus medius muscle, because of its role in controlling pelvic stability in the frontal plane ${ }^{17}$.

Part of the therapy is focused on regaining proper neuromuscular pattern, for which treatment considers exercises aimed at the activation of the gluteus medius with no further activation of the iliopsoas. Intramuscular electromyography studies measure the activation of the gluteus medius versus the iliopsoas during these exercises, and their results show that exercises like single-leg bridge, prone heel squeeze (external rotation isometrics) and side-lying hip abduction present the greatest activity of the gluteus medius and the least of the iliopsoas ${ }^{18}$.

Lastly one point considered in this phase, and cer- tainly one of greater interest for subjects, relates to establishing independent gait. Gait with no technical assistance occurs when the surgeon approves the removal of canes in order to normalize gait and achieve basic needs of daily life, such as going up or down stairs.

Thus, the main objectives include the ceasing of dependence of canes, normalizing gait and maintaining neuromuscular control during daily life activities (DLA) of weight-bearing nature, through restoration of complete passive and active mobility. In order to achieve this goal, we establish operational objectives to improve gait, such as increasing strength of the gluteus maximus, gluteus medius, iliopsoas and deep rotator muscles, which can be observed in the progressive improvement in postural control in both single leg and double leg support phases ${ }^{19}$.

Caution: it is important to consider errors that may occur during this rehabilitation phase; negligence in treatment can trigger situations that hinder recovery.

One of these situations is the unnecessary stress on hip flexors and internal rotators, which occurs mainly as a result of performing exercises beyond protected ranges. That is why one measure to apply during therapy is to prescribe extension exercises of hip until neutral position, and using a night abductor pillow up to the third week $^{20}$.

Another common cause that may hinder this phase is the early discontinuation of the use of walking canes; that is why treatment considers gait using two canes until the 2-3 week, and removing the second cane at the 3-4 week or according to medical indication ${ }^{21}$

Poor hip stability during movement may also lead to excessive compressive forces through the hip joint ${ }^{22}$.

In order to move to the next phase, the following goals should have been achieved:

1. minimum pain with phase 1 exercises;

2. ROM increase;

3. proper muscle activation in all exercises;

4. removal of canes.

\section{Phase 2}

\section{Neuromuscular control phase (4-8 weeks post- surgery)}

The general objective of this phase is achieving daily life activities (DLA) independently and pain-free. Restoring antalgic independent gait is one of the main goals for both for the therapist and the subject, for which active and passive joint mobility ranges must be achieved, which are necessary for normal gait. Manual techniques include neuromuscular inhibition, mobilization of soft tissues, and stretching should be used to minimize muscle tone ${ }^{16}$.

Improving neuromuscular control and re-training muscle activation timing, in addition to the beginning of muscle strength exercises mainly for the gluteus medius and gluteus maximus, will be a constant during this period.

Control of the frontal plane of hip and pelvis must be quickly reestablished in order to restore functional gait. During the support phase, hip abductor muscles, particularly the gluteus medius, initially works eccen- 
Comparative analysis of kinesiotherapy rehabilitation after hip arthroscopy, quantified by harris and vail hip scores: a retrospective study

trically in the control of the contralateral pelvic drop. The concentric action of hip abductors rises the contralateral pelvis during the support phase in order to lift the body's center of mass to its higher point during gait ${ }^{23}$, thus facilitating gait to a great extent and avoiding difficulties in walking or functional impotency.

Passive motion exercises instructed by the therapist must be focused on reestablishing functional ranges; therefore, special emphasis is given to managing soft tissues at the capsular and muscular level; the iliopsoas, TFL and abductors are affected by this hyperactivity pattern and get shortened, which has been proven by muscle length tests (such as Thomas' test, among others) ${ }^{16}$.

Manual therapy also includes anterior to posterior graded mobilization stretching, and even some type of long-axis distraction in order to reset neuromuscular activity ${ }^{24}$.

If the iliopsoas muscle is hyperactive it does not mean that it is an efficient muscle; that is why it must be re-trained without overloading it. One of the procedures to evaluate the iliopsoas' function is to test the ability to flex the affected hip through the performance of 10 active hip flexion repetitions, starting from $20^{\circ}$ of hip extension, to $90^{\circ}$ of flexion. $20^{\circ}$ extension is used to assess the muscle's ability to initiate swing during gait, up to $90^{\circ}$ of flexion in order to assess the functional ability to climb stairs and perform pre-athletic movements ${ }^{19}$. It is important to avoid overloads at all times, as this could lead to possible irritations hindering rehabilitation.

Caution: like in Phase 1, irritation of hip flexors and abductors must be prevented, as well as joint overloads, and ballistic or aggressive stretching ${ }^{21}$.

Progression criteria:

1. pain-free normal gait;

2. complete ROM;

3. absence of joint inflammation, muscle irritation or pain;

4. proper neuromuscular control in functional activities.

\section{Phase 3}

Strength, resistance and functional movement patterns (8-12 weeks)

Phase 3 usually starts between the 6th and 8th week post-surgery depending on the subject, and its objective is based on the further restoration of muscle resistance and strength; improve cardiovascular condition, optimize neuromuscular control, balance and proprioception ${ }^{25}$. Neuromuscular control and gait are in a state of marked improvement, and the emphasis is made on the improvement of concentric control in the sagittal and frontal plane by the subject, and otherwise eccentric work is focused on the transverse plane ${ }^{19}$. This phase aims to improve cardiovascular condition and the subject's ability to perform agility exercises, seeking pre-injury levels of performance ${ }^{25}$. In order to achieve this, the subject must be able to tolerate single-leg loads properly and pain free, as well as eccentric work, and if the subject requires it, perform activities that are directly related to a sport 26 . The subject must show good tolerance to ambulation in order to improve tolerance and progress to sportspecific tasks. The tasks must be carried out within ranges between 0-1 in Borg's perceived exertion scale 27 .

The load and volume of increase in DLA, ambulation and specific sport functional exercises must be supervised and carefully managed beyond rehabilitation; the timing between stages is highly variable.

Caution: avoid ballistic-type movements, not include yet the use of treadmill gait, and as in the other recovery phases, prevent irritation of hip flexors and avoid exercises that involve contact or high speed at the beginning of the sport phase.

Progression criteria:

1. the subject performs all exercises from phase 3 without pain and in a correct way;

2. cardiovascular capacity similar to pre-surgery state;

3. move to Sport Hip Test only under medical indication.

\section{Phase 4}

Return to sport activities

Phase 4 has the objective of returning to play sports $^{26}$.

This phase usually begins between 8 to 16 weeks, depending on the subject's status ${ }^{6}$.

Recommended criteria to move to the free activity Phase 4 include:

1. confirm that hip flexion strength is higher than $85 \%$ with respect to the healthy side;

2. ROM must be complete and pain free;

3. the subject must be able to perform specific sport exercises at full speed and complete them successfully ${ }^{24}$.

During this phase, the subject continues to perform Phase 3 muscle-strengthening activities with an increase in loads and exercise time. Once the subject starts his or her specific sport activity, it is important to ensure that the subject is able to perform low level plyometric exercises (e.g., one-sided half squats), multidirectional agility drills (e.g., ladder drills and lateral movements at high speed) and circuit training. Available variables in the exercise include speed of movement, planes of motion, and rest intervals ${ }^{19}$.

Caution: this phase considers the inclusion of all pain free activities. One of the only aspects to consider is that impact exercises like jogging are recommended until 2 weeks after this phase.

\section{Harris Hip score}

The Harris Hip score (HHS) is a multidimensional observational scale which includes different assessment items such as pain, physical function, deformity, and range of motion ${ }^{10,28}$. The scale has a 100-point maximum divided based on assessed items: the item of pain has a score of 44 points; physical function has 7 items with a total of 47 points; deformity has 5 items with a total of 5 points; and range of motion has 5 items, with a total of 4 points $^{9,28}$. The scale has a total 
Table II. This Table shows the mean value and S.D. of the Harris Hip Score (HHS) and Vail score, and the number of patients who complete the different sessions of the rehabilitation program (1: the first session of rehabilitation; 10: ten sessions; 20: twenty sessions).

\begin{tabular}{llllll}
\hline & No. of subjects & HHS & Vail Score & & \\
\hline & & Mean & Standard dev. & Mean & Standard dev. \\
\hline session 1 & 100 & 60.539 & 79.228 & 45.586 & 15.389 \\
\hline session 10 & 96 & 75.596 & 15.217 & 67.155 & 16.357 \\
\hline session 20 & 50 & 86.988 & 12.582 & 76.604 & 16.091 \\
\hline
\end{tabular}

of 100 points, in which the traditional categorization is as follows: $0-70$ is considered poor; $70-79$ is considered fair; $80-89$ is considered good; and $90-100$ is considered excellent ${ }^{9,28}$

\section{Vail Hip score}

The Vail Hip score (VHS) is a sensitive scale to assess subjects who underwent hip arthroscopies ${ }^{12}$; it includes 10 questions focused on pain, stiffness, gait and function ${ }^{19}$

The scale has a maximum of 100 points divided based on the assessed items: pain includes three questions with a total of 50 points; stiffness includes two questions with a total of 20 points; gait includes one question with a total of 5 points, and functions include four questions with a total of 25 points $^{19}$

Total score amounts to 100 points, and the Spanishtranslated VHS has a high construct validity ${ }^{12}$.

\section{Procedure}

We accessed an Excel database of self-report scales of participants who entered rehabilitation after hip arthroscopy in our institution's Center for Sports Medicine in Chile, between 2011 and 2016. During the first kinesiology session an initial evaluation was performed; such evaluation included the set of two selfreport scales (HHS and VHS) attached to the standard form. Once the therapist filled the form, he gave both scores to participants for he or she to fill them at the beginning of therapy.

After the first 10 kinesiotherapy sessions, the therapist in charge reassessed both scales in order to continue with the subsequent ten kinesiotherapy sessions, which occurs during the 4th and 5 th week postsurgery; this said, and based on the individual development of each subject, the therapy is carried out according to the standardized rehabilitation protocol validated by our institution's Center for Sports Medicine; at the end of the second round of ten kinesiotherapy sessions, the set of self-report scales was reassessed.

\section{Statistical analysis}

Software SPSS 15.0 (SPSS 15.0 for Windows) was used for the statistical analysis. The repeated mea- sures ANOVA test was used to compare data variance, together with a Bonferroni post-hoc test. Significance level for all statistical tests was 0.01 .

\section{Results}

The review of the database showed that a total of 102 subjects who underwent hip arthroscopy surgery completed the self-report scales in any of the three occasions; a total of 100 subjects completed the scales in the first session; 96 subjects completed the scales in the 10th session, and 50 completed the scales in the 20th session. Table II shows the mean and standard deviation of each scale's data.

A total of 48 subjects were ultimately considered in the study, as they completed the scales on the three occasions; Table III shows the mean and standard deviation of each score's data. The multivariate contrast analysis shows an exact level of significance $F=147.420 ; p=0.000$ in the 4 tests of the HHS effect; this means that the difference in scores obtained in each evaluation is significant. The multivariate contrast analysis shows an exact level of significance $F=82.160 ; p=0.000$ in the 4 tests of the VHS effect; this means that the difference in scores obtained in each evaluation is significant.

Mauchly's sphericity test result scores: the HHS scale showed significant differences of $W=0.722 ; p=0.001$; the VHS scale showed significant differences of $\mathrm{W}=0.830 ; \mathrm{p}=0.014$. This means there is a significant

Table III. Into this Table the mean value and S.D. of the Harris Hip Score (HHS) and Vail score of the patients included into the study are reported (1: the first session of rehabilitation; 10: ten sessions; 20: twenty sessions).

\section{Mean and standard dev.; answers to 3 Scales}

\begin{tabular}{lll}
\hline & Mean & Standard dev. \\
\hline HHS 1 & 49.339 & 17.320 \\
\hline HHS 10 & 71.497 & 15.883 \\
\hline HHS 20 & 87.433 & 12.005 \\
\hline Vail Score 1 & 45.75 & 14.810 \\
\hline Vail Score 10 & 62.895 & 16.214 \\
\hline Vail Score 20 & 76.358 & 16.375 \\
\hline
\end{tabular}


Comparative analysis of kinesiotherapy rehabilitation after hip arthroscopy, quantified by harris and vail hip scores: a retrospective study

effect in HHS and VHS scales between the start of therapy, the 10th session and the 20th session.

With respect to the HHS scale, the within-subject effect shows exact significant differences; therefore it can be concluded that scores are not the same between the evaluations of such scale; statistics of the four tests show exact statistics $F=169.451 ; p=0.000$. With respect to the VHS scale, the within-subject effect shows exact significant differences $F=115.387$; $p=0.000$ in the four tests; therefore it can be concluded that scores are not the same between the evaluations of such scale.

The analysis of estimated marginal measurements through Bonferroni test showed an exact statistic $p=0.000$ for HHS in all comparisons; and an exact statistic $\mathrm{p}=0.000$ for VHS in all comparisons.

\section{Discussion}

Arthroscopy surgery for hip impingement has acquired high effectiveness as therapeutic approach to decrease symptoms in people with hip pain and improve joint ranges of motion ${ }^{19-22,24}$. Our institution's physical therapy rehabilitation protocol in people who underwent surgery for femoroacetabular impingement is standardized and validated by literature previously which has been tested in biomedical studies $14,17,19,21,24,26$. This is why this study compared the Excel score database of HHS and VHS scales made between 2011 and 2016; before starting kinesiotherapy and every ten sessions of progression, in order to quantify the advance or the existing difference through the kinesiotherapy rehabilitation process. The main results show statistically significant differences in the statistical analysis scores, which showed exact significant differences in their measurement of multivariate contrasts and significant differences in data sphericity; in addition, the within-subject effect showed exact significant differences in the evaluation.

This may be because our institution's kinesiotherapy, which is carried out based on a post hip arthroscopy rehabilitation protocol, is effective in form and execution for its participants. It is worth mentioning the importance of quantification and the input of the scales between 10-session terms, which allowed us to quantify the differences within subjects who fully completed kinesiotherapy rehabilitation in our institution.

The study of Grysbok et al. (2015) ${ }^{29}$ reviewed different rehabilitation protocols, and its results suggested that post-surgery rehabilitation for hip impingement had a poor heterogeneity of significant results as therapeutic protocol, and poor frequency of progress reports. This contrasts with the case of our institution's protocol, as we used two valid and reliable selfreport tools which we evaluated in 10-session intervals, since the start of rehabilitation until medical discharge.

Even though our institution's protocol has a structure at every phase of kinesiotherapy rehabilitation, it is important to mention that it is customized based on each subject's progress and DLA needs, because an inflexible rehabilitation program limits individual adaptation and subjects' adherence to a program ${ }^{30}$ It is always important to emphasize on the healing process in the rehabilitation phase, and progress be based on the progression criteria to the following phase ${ }^{24}$, all of this aimed at achieving a functional rehabilitation of what a patient needs to return to normal DLA based on his or her needs.

This study reviewed the database of subjects who completed the HHS and VHS scales and entered kinesiotherapy rehabilitation between 2011-2016, and a high significance of results in each evaluation phase was observed. It is worth mentioning that such database included a high loss of subjects who failed to answer the three phases of evaluation, which can be inferred as lack of adherence to the rehabilitation program, which in turn can be influenced by unspecified multiple factors. Thus, for further studies it is important to emphasize on this matter to the subject and the kinesics rehabilitation team: encourage subjects to complete treatment until the last phase of rehabilitation, and continue completing the scales, as they have proven to be useful tools in quantifying participants' progress.

The results of the comparative analysis can help determine a cause-effect relationship between variables, as this type of study compared the self-report scales used in an average proportion of time which was previously specified. However, it is important to mention that the study provides a partial explanation; there are other variables which must be assessed and which could be included in further studies, as there may be other factors affecting the evaluations performed and the results of each questionnaire. Although from an observational perspective study, we cannot state that subjects who did not completed kinesiotherapy rehabilitation in our institution obtain bad results, it is evident that those subjects who completed their rehabilitation in all phases in our institution had statistically significant improvements.

This study was designed according the ethical standards of the journal ${ }^{31}$

\section{Conclusion}

This study found significant differences in the measurement of self-report scales (HHS and VHS) on the three occasions of evaluation during the kinesiotherapy rehabilitation process. Current literature provides little evidence on kinesics rehabilitation protocols and their effectiveness; this said, this study offers our institution's Center for Sports Medicine kinesiotherapy rehabilitation protocol for use as guideline, as it is based on a systematic and individualized progression of a subject's needs to achieve balance and proper rehabilitation. In addition, the use of HHS and VHS self-report scales is recommended to quantify the progress of subjects who had undergone hip arthroscopy for femoroacetabular impingement. 


\section{References}

1. Lequesne $M$, Belläche $L$. Anterior femoroacetabular impingement: An update. Joint Bone Spine. 2012;79(3):249-55.

2. Mardones R, Barrientos V, Nemtala F, et al. Femoroacetabular impingement as cause of inguinal pain. Rev Med Chile. 2010;138:102-8

3. Tijssen M, van Cingel R, van Melick N, et al. Patient-Reported outcome questionnaires for hip arthroscopy: a systematic review of the psychometric evidence. BMC Musculoskeletal Disorders. 2011;12:117.

4. Nielsen T, Miller L, Lund B, et al. Outcome of arthroscopic treatment for symptomatic femoroacetabular impiggement. BMC Musculoskeletal Disorders. 2014;15:394.

5. Tijssen M, van Cingel R, Willemsen L, de Visser E. Diagnostics of Femoroacetabular Impingement and Labral Pathology of the Hip: A systematic Review of the Accuracy and Validity of Physical Test. Arthroscopy: The journal of Arthroscopic and Relatd Surgery. 2012;28(6):860-71.

6. Wahoff M, Ryan M. Rehabilitation After Hip Femoroacetabular Impingement Arthroscopy. Clin Sports Med. 2011;30 (2):463-82.

7. Cheatham S, Kolber M. Rehabilitation after hip Arthroscopy and Labral repair in a high School Football Athlete. Case report. IJSPT. 2012;7(2):173-84

8. Mohamed N, Arndt D, McGrory B, et al. The Harris Hip Score. Comparison of Patient Self-report With Surgeon Assessment. The Journal of Arthroplasty. 2001;16(5):575-80

9. Singh J, Schleck C, Harmsen S, et al. Clinically important improvement thresholds for Harris Hip Score and its ability to predict revision risk after primary total hip arthroplasty. BMC Musculoskeletal Disorders. 2016;17:256.

10. Hoeksma H, van den Ende $\mathrm{C}$, Ronday $\mathrm{H}$, et al. Comparison of the responsiveness of the Harris Hip Score with generic measures for hip function in osteoarthritis if the hip. Ann Rheum Dis. 2003;62:935-38.

11. Edwards P, Queen R, Butler R, et al. Are Range of Motion Measurements Needed When Calculating the Harris Hip Score? The Journal of Arthroplasty xxx. 2015;1- 5.

12. Mardones R et al. Which is the Most Reliable Spanish Translated Score for Hip Arthroscopy? Arthroscopy. 2013;29(12): 188-9.

13. Malloy P, Malloy M, Draovitch P. Guidelines and pitfalls for the rehabilitation following hip arthroscopy. Curr Rev Musculoskelet Med. 2013;6(3):235-24.

14. Cantu R. Soft tissue healing considerations after surgery, in Maxey LM(ed): Rehabilitation for the Postsurgical Orthopedic Patient. St Louis, MO, Mosby, 2001.

15. Jobe FW, Glousman RE. Anterior capsulolabral reconstruction, in Pau-los LE, Tibone JE (eds): Operative Technique in Shoulder SurgeryGaithersburg, MD, Aspen Publishers, 1992.

16. Beck M. Groin Pain after Open FAI Surgery: The Role of In- traarticular Adhesions. Clin Orthop Relat Res. 2009;467(3): 769-74.

17. Neumann DA. Biomechanical analysis of selected principles of hip joint protection. Arthritis Care Res. 1989;2(4):146-55.

18. Philippon MJ, Decker MJ, Giphart JE, et al. Rehabilitation exercise progression for the gluteus medius muscle with consideration for iliopsoas tendinitis: an in vivo electromyography study. Am J Sports Med. 2011;39(8):1777-85.

19. Wahoff M, Dischiavi S, Hodge J, et al. Rehabilitation after labral repair and femoroacetabular decompression: CriteriaBased progression through the return to sport phase. Int $J$ Sports Phys Ther. 2014;9(6):813-26.

20. Lewis CL, Sahrmann SA, Moran DW. Anterior hip joint forces increases with hip extension, decreased gluteal force or decreased iliopsoas force. J Biomech. 2007;40(16):3725-31.

21. Bolgla LA. Electromyographic analysis of hip rehabilitation exercises in a group of healthy subjects. J Orthop Sports Phys Ther. 2005;35(8):487-94.

22. Malloy P, Gray K, Wolff AB.Rehabilitation After Hip Arthroscopy: A Movement Control-Based Perspective. Clin Sports Med. 2016;35(3):503-21.

23. Simoneau GG. Kinesiology of walking. In: Neumann D, editor. Kinesiology of the musculoskeletal system: foundations for rehabilitation. 2. St. Louis: Mosby Elsevier

24. Cheatham S. Rehabilitation after hip arthroscopy and labral repair in a High School football athlete Int J Sports Phys Ther. 2012;7(2):173-84.

25. Garrison JC, Osler MT, Singleton SB. Rehabilitation after arthroscopy of an acetabular labral tear. N Am J Sports Phys Ther. 2007;2(4):241-50.

26. Pierce CM. Ice hockey goaltender rehabilitation, including onice progression, after arthroscopic hip surgery for femoroacetabular impingement. J Orthop Sports Phys Ther. 2013;43 (3):129-41.

27. Scherr J, Wolfarth B, Christle JW, et al. Associations between Borg's rating of perceived exertion and physiological measures of exercise intensity. Eur J Appl Physiol. 2013;113(1): 147-55.

28. Guimarães RP, Alves DP, Azuaga TL, et al. Translation and transcultural adaptation of the modified harris hip score. Acta Ortop Bras. 2010;18(6):339-42.

29. Grzybowski J, Malloy P, Stegemann C, et al. Rehabilitation Following Hip Arthroscopy -A Systematic Review. Front Surg. 2015;2(21):1-10.

30. Bennell K, Donnell J, Takla A, et al. Efficacy of a physiotherapy rehabilitation program for individuals undergoing arthroscopic management of femoroacetabular impingement-the FAIR trial: a randomised controlled trial protocol. BMC Musculoskeletal Disorders. 2014;15(58):1-11.

31. Padulo J, Oliva F, Frizziero A, Maffulli N. Muscles, Ligaments and Tendons Journal. Basic principles and recommendations in clinical and field science research: 2016 Update. MLTJ. 2016;6(1):1-5. 